\title{
TWISTED BURNSIDE THEOREM FOR TWO-STEP TORSION-FREE NILPOTENT GROUPS
}

\author{
ALEXANDER FEL'SHTYN, FEDOR INDUKAEV, AND EVGENIJ TROITSKY
}

\begin{abstract}
It is proved that the Reidemeister number of any automorphism of any finitely generated torsion-free two-step nilpotent group coincides with the number of fixed points of the corresponding homeomorphism of the subspace of the unitary dual related to finite-dimensional representations, if one of these numbers is finite. An important example of the discrete Heisenberg group is studied in detail.
\end{abstract}

\section{Contents}

1. Introduction

2. Preliminary considerations

3. Extensions and Reidemeister classes

4. Torsion-free two-step nilpotent groups 4

5. Twisted Burnside theorem

6. An infinite series of automorphisms of discrete Heisenberg group

7. Finding of fixed representations

References

\section{INTRODUCTION}

Definition 1.1. Let $G$ be a countable discrete group and $\phi: G \rightarrow G$ an endomorphism. Two elements $x, x^{\prime} \in G$ are said to be $\phi$-conjugate or twisted conjugate, iff there exists $g \in G$ with

$$
x^{\prime}=g x \phi\left(g^{-1}\right) .
$$

We write $\{x\}_{\phi}$ for the $\phi$-conjugacy or twisted conjugacy class of the element $x \in G$. The number of $\phi$-conjugacy classes is called the Reidemeister number of an endomorphism $\phi$ and is denoted by $R(\phi)$. If $\phi$ is the identity map then the $\phi$-conjugacy classes are the usual conjugacy classes in the group $G$.

If $G$ is a finite group, then the classical Burnside theorem (see e.g. [13, p. 140]) says that the number of classes of irreducible representations is equal to the number of conjugacy classes of elements of $G$. Let $\widehat{G}$ be the unitary dual of $G$, i.e. the set of equivalence classes of unitary irreducible representations of $G$.

2000 Mathematics Subject Classification. 20C; 20E45; 22D10; 22D25; 22D30; 37C25; 43A30; 46L.

Key words and phrases. Reidemeister number, twisted conjugacy classes, Burnside theorem, two-step nilpotent group, Heisenberg group.

The third author is partially supported by RFFI Grant 05-01-00923, Grant for the support of leading scientific schools and Grant "Universities of Russia" YP.04.02.530. 
Therefore, by the Burnside's theorem, if $\phi$ is the identity automorphism of any finite group $G$, then we have $R(\phi)=\# \operatorname{Fix}(\widehat{\phi})$.

One of the main achievements in the field till now is the following result.

Theorem $1.2([5])$. Let $G$ be a finitely generated discrete group of type I, $\phi$ one of its endomorphism, $R(\phi)$ the number of $\phi$-conjugacy classes, and $S(\phi)=\# \operatorname{Fix}(\widehat{\phi})$ the number of $\widehat{\phi}$-invariant equivalence classes of irreducible unitary representations. If one of $R(\phi)$ and $S(\phi)$ is finite, then it is equal to the other.

The research is motivated not only by a natural desire to extend the classical Burnside theorem to the case of infinite groups and twisted conjugacy classes, but also by dynamical applications. Namely, the identification of Reidemeister number with a number of fixed points in a natural way, has some very interesting consequences in Dynamics and Number Theory (see [5]).

On the other hand, one can introduce the number $R_{*}(\phi)$ of "Reidemeister classes related to twisted invariant functions on $G$ from the Fourier-Stieltjes algebra $B(G)$ ", or more precisely, the dimension of the space of twisted invariant functions on $G$ which can be extended up to bounded functionals on the group algebra $C^{*}(G)$. Let $S_{*}(\phi)$ be the sum of codimensions of subspaces $L_{I} \subset C^{*}(G) / I$, where $L_{I}$ is generated by elements of the form $a-L_{g} a L_{\phi\left(g^{-1}\right)}$ and $I$ runs over the Glimm spectrum of $G$, i. e. on the complete regularization of $\widehat{G}$. We call $S_{*}(\phi)$ the number of generalized fixed points of $\widehat{\phi}$ on the Glimm spectrum of $G$.

Theorem 1.3 (weak twisted Burnside theorem, [15]). The number $R_{*}(\phi)$ is equal to the number $S_{*}(\phi)$ of generalized fixed points of $\widehat{\phi}$ on the Glimm spectrum of $G$, if one of $R_{*}(\phi)$ and $S_{*}(\phi)$ is finite.

This result allows to obtain the strong form of twisted Burnside theorem $R(\phi)=S(\phi)$ in a number of cases.

The interest in twisted conjugacy relations has its origins, in particular, in the NielsenReidemeister fixed point theory (see, e.g. [12, 4]), in Selberg theory (see, eg. [14, 1]), and Algebraic Geometry (see, e.g. [10]).

The congruences give some necessary conditions for the realization problem for Reidemeister numbers in topological dynamics.

Let us remark that it is known that the Reidemeister number of an endomorphism of a finitely generated Abelian group is finite iff 1 is not in the spectrum of the restriction of this endomorphism to the free part of the group (see, e.g. [12]). The Reidemeister number is infinite for any automorphism of a non-elementary Gromov hyperbolic group [7].

The main results of the present paper are as follows.

- The Reidemeister number of any automorphism of any finitely generated torsionfree two-step nilpotent group coincides with the number of fixed points of the corresponding homeomorphism of the subspace of the unitary dual related to finitedimensional representations, if one of these numbers is finite.

- For the discrete Heisenberg group and any even number $2 N$ an automorphism $\phi$ is constructed with $R(\phi)=2 N$.

- For $N=1$ the mentioned fixed points are found explicitly. 
Acknowledgement. The present research is a part of joint research programm of A. Fel'shtyn and E. Troitsky in Max-Planck-Institut für Mathematik (MPI) in Bonn. We would like to thank the MPI for its kind support and hospitality while the most part of this work has been completed.

The authors are grateful to V. Balantsev, M. B. Bekka, R. Hill, V. Manuilov, A. Mishchenko, A. Shtern, L. Vainerman, A. Vershik for helpful discussions.

The results of Sections 2 and 3 are obtained by A. Fel'shtyn and E. Troitsky, the results of Sections 4 and 5 are obtained by E. Troitsky (in more detail and for a much more general case they appear in [6]), the results of Sections 6 and 7 are obtained by F. Indukaev.

\section{Preliminary CONSIDERATions}

Lemma 2.1. Let $G$ be abelian. The twisted conjugacy class $H$ of $e$ is a subgroup. The other ones are cosets $g H$.

Proof. The first statement follows from the equalities

$$
h \phi\left(h^{-1}\right) g \phi\left(g^{-1}\right)=g h \phi\left((g h)^{-1}, \quad\left(h \phi\left(h^{-1}\right)\right)^{-1}=\phi(h) h^{-1}=h^{-1} \phi(h) .\right.
$$

For the second statement suppose $a \sim b$, i.e. $b=h a \phi\left(h^{-1}\right)$. Then

$$
g b=g h a \phi\left(h^{-1}\right)=h(g a) \phi\left(h^{-1}\right), \quad g b \sim g a .
$$

Lemma $2.2([4,12])$. An automorphism $\phi: \mathbb{Z}^{k} \rightarrow \mathbb{Z}^{k}$ with $R(\phi)<\infty$ has a unique fixed point: identity element.

Let us denote by $\tau_{g}: G \rightarrow G$ the automorphism $\tau_{g}(\widetilde{g})=g \widetilde{g} \phi\left(g^{-1}\right)$ for $g \in G$. Its restriction on a normal subgroup we will denote by $\tau_{g}$ as well.

Lemma 2.3. $\{g\}_{\phi} k=\{g k\}_{\tau_{k-1} \circ \phi}$.

Proof. Let $g^{\prime}=f g \phi\left(f^{-1}\right)$ be $\phi$-conjugate to $g$. Then

$$
g^{\prime} k=f g \phi\left(f^{-1}\right) k=f g k k^{-1} \phi\left(f^{-1}\right) k=f(g k)\left(\tau_{k^{-1}} \circ \phi\right)\left(f^{-1}\right) .
$$

Conversely, if $g^{\prime}$ is $\tau_{k^{-1}} \circ \phi$-conjugate to $g$, then

$$
g^{\prime} k^{-1}=f g\left(\tau_{k^{-1}} \circ \phi\right)\left(f^{-1}\right) k^{-1}=f g k^{-1} \phi\left(f^{-1}\right) .
$$

Hence a shift maps $\phi$-conjugacy classes onto classes related to another automorphism.

\section{Extensions And Reidemeister Classes}

Consider a group extension respecting homomorphism $\phi$ :

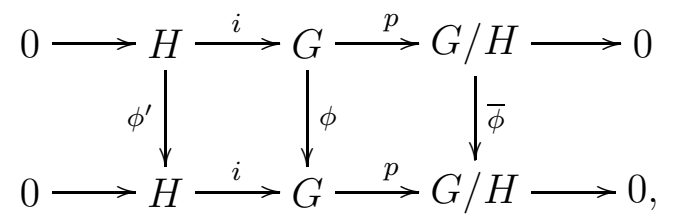

where $H$ is a normal subgroup of $G$. The following argument has partial intersection with $[8,9]$. 
First of all let us notice that the Reidemeister classes of $\phi$ in $G$ are mapped epimorphically on classes of $\bar{\phi}$ in $G / H$. Indeed,

$$
p(\widetilde{g}) p(g) \bar{\phi}\left(p\left(\widetilde{g}^{-1}\right)\right)=p\left(\widetilde{g} g \phi\left(\widetilde{g}^{-1}\right) .\right.
$$

Suppose, $R(\phi)<\infty$. Then the previous remark implies $R(\bar{\phi})<\infty$. Consider a class $K=\{h\}_{\tau_{g} \phi^{\prime}}$, where $\tau_{g}(h):=g h g^{-1}, g \in G, h \in H$. The corresponding equivalence relation is

$$
h \sim \widetilde{h} h g \phi^{\prime}\left(\widetilde{h}^{-1}\right) g^{-1} .
$$

Since $H$ is normal, the automorphism $\tau_{g}: H \rightarrow H$ is well defined. We will denote by $K$ the image $i K$ as well. By (3) the shift $K g$ is a subset of $H g$ is characterized by

$$
h g \sim \widetilde{h}(h g) \phi^{\prime}\left(\widetilde{h}^{-1}\right) .
$$

Hence it is a subset of $\{h g\}_{\phi} \cap H g$ and the partition $H g=\cup\left(\{h\}_{\tau_{g} \phi^{\prime}}\right) g$ is a subpartition of $H g=\cup\left(H g \cap\{h g\}_{\phi}\right)$.

Lemma $3.1([8])$. Suppose, \# $\operatorname{Fix}\left(\tau_{z} \bar{\phi}\right)=1$ for some representative $z$ of any class $\{y\}_{\bar{\phi}}$. Let $\left\{z_{\alpha}\right\}$ be the full collection of these representatives and $g_{\alpha}$ some elements of $G$ such that $p\left(g_{\alpha}\right)=z_{\alpha}$. If $R(\phi)<\infty$, then

$$
R(\phi)=\sum_{\alpha} R\left(\tau_{g_{\alpha}} \phi^{\prime}\right)
$$

\section{TORSION-FREE TWO-STEP NILPOTENT GROUPS}

A torsion-free f.g. two-step nilpotent group $G$ is an extension (1) with $H \cong \mathbb{Z}^{m}$, $G / H \cong \mathbb{Z}^{k}$, and $H$ being the center of $G$. Since $H$ is the center, the extension respects any $\phi$.

Theorem 4.1. Let $\phi$ be an automorphism of a torsion-free f.g. two-step nilpotent group $G$ and $R(\phi)<\infty$. Then all $\phi$-class functions are coefficients of finite-dimensional representations of $G$.

Proof. By Lemma 2.2 and Lemma 3.1 one has $R\left(\phi^{\prime}\right)<\infty$. Taking a quotient by subgroup $H_{1}=\{e\}_{\phi^{\prime}}$ (see Lemma 2.1) one obtains a factor group $G_{1}=G / H_{1}$ with a bijection of $\phi$-conjugacy classes under the projection. This means that it is sufficient to prove the statement for $G_{1}$. For $G_{1}$ we have the following $\phi$-invariant extension:

$$
H / H_{1}=A \rightarrow G_{1} \rightarrow \mathbb{Z}^{k}
$$

where the abelian group $A$ is finite.

By Lemma 2.3 the number of different sets among the shifts of a $\phi$-conjugacy class is less or equal $R(\phi) \times M$, where $M$ is the number of different automorphisms $\tau_{g}: G_{1} \rightarrow G_{1}$. From the facts that $G_{1}$ is finitely generated, $A$ is finite, and $G_{1} / A$ is abelian, it follows that $M<\infty$. Hence the common stabilizer of classes has a finite index. Let $S$ be a characteristic subgroup of finite index inside this stabilizer. Then a characteristic function of $\phi$-conjugacy class is an inverse image under projection of a function on the finite group $G_{1} / S$. This function is a coefficient of a finite-dimensional representation $\rho: G_{1} / S \rightarrow$ End $V$. Then the characteristic function of the original set is the corresponding coefficient of the representation

$$
G \rightarrow G / H=G_{1} \rightarrow G_{1} / S \stackrel{\rho}{\longrightarrow} \text { End } V .
$$




\section{Twisted Burnside THEOREM}

Definition 5.1. Denote by $\widehat{G}_{f}$ the subset of the unitary dual $\widehat{G}$ related to finite-dimensional representations.

Theorem 5.2. Let $G$ be a torsion-free f.g. two-step nilpotent group and $\phi$ its automorphism. Denote by $S_{f}(\phi)$ the number of fixed points of $\widehat{\phi}_{f}$ on $\widehat{G}_{f}$. Then

$$
R(\phi)=S_{f}(\phi)
$$

if one of this numbers is finite.

Proof. Let us start from the following observation. Let $\Sigma$ be the universal compact group associated with $G$ and $\alpha: G \rightarrow \Sigma$ the canonical morphism (see, e.g. [3, Sect. 16.1]). Then $\widehat{G}_{f}=\widehat{\Sigma}[3,16.1 .3]$. The coefficients of (finite-dimensional) non-equivalent irreducible representations of $\Sigma$ are linear independent by Peter-Weyl theorem as functions on $\Sigma$. Hence the corresponding functions on $G$ are linearly independent.

It is sufficient to verify the following three statements:

1) If $R(\phi)<\infty$, than each $\phi$-class function is a finite linear combination of twistedinvariant functionals being coefficients of points of Fix $\widehat{\phi}_{f}$.

2) If $\rho \in$ Fix $\widehat{\phi}_{f}$, there exists one and only one (up to scaling) twisted invariant functional on $\rho\left(C^{*}(G)\right)$ (this is a finite full matrix algebra).

3) For different $\rho$ the corresponding $\phi$-class functions are linearly independent. This follows from the remark at the beginning of the proof.

Let us remark that Theorem 4.1 implies in particular that $\phi$-central functions (for $\phi$ with $R(\phi)<\infty)$ are functionals on $C^{*}(G)$, not only $L^{1}(G)$, i.e. are in the Fourier-Stieltijes algebra $B(G)$.

The statement 1) follows from Theorem 4.1. Indeed, the twisted action takes functional related to some representation to another functional related with the same representation. Since they are linearly independent, this component of the linear combination has to be twisted-invariant. For any $\rho \in \widehat{G}_{f}$ any functional has the form $a \mapsto \operatorname{Tr}(b a)$ for some fixed $b$. Twisted invariance implies twisted invariance of $b$ (evident details can be found in $[5$, Sect. 3]). Hence, $b$ is intertwining between $\rho$ and $\rho \circ \phi$ and $\rho \in \operatorname{Fix}\left(\widehat{\phi}_{f}\right)$. The uniqueness of intertwining operator (up to scaling) implies 2).

\section{An infinite SERIES of Automorphisms of Discrete Heisenberg group}

Consider the set of matrices

$$
\left(\begin{array}{ccc}
1 & k & m \\
0 & 1 & l \\
0 & 0 & 1
\end{array}\right), \text { where } k, l, m \in \mathbb{Z}
$$

They form the discrete Heisenberg group $G$ with respect to the matrix multiplication. This group has 3 generators:

$$
a=\left(\begin{array}{ccc}
1 & 1 & 0 \\
0 & 1 & 0 \\
0 & 0 & 1
\end{array}\right) ; \quad b=\left(\begin{array}{ccc}
1 & 0 & 0 \\
0 & 1 & 1 \\
0 & 0 & 1
\end{array}\right) ; \quad c=\left(\begin{array}{ccc}
1 & 0 & 1 \\
0 & 1 & 0 \\
0 & 0 & 1
\end{array}\right)
$$


and

$$
\left(\begin{array}{ccc}
1 & k & m \\
0 & 1 & l \\
0 & 0 & 1
\end{array}\right)=b^{l} a^{k} c^{m} .
$$

The relations are the following

$$
[a, c]=e ; \quad[b, c]=e ; \quad[a, b]=c .
$$

We will denote elements of $G$ as triples:

$$
\left(\begin{array}{ccc}
1 & k & m \\
0 & 1 & l \\
0 & 0 & 1
\end{array}\right)=(k, l, m)
$$

in particular,

$$
a=(1,0,0) ; \quad b=(0,1,0) ; \quad c=(0,0,1) .
$$

The (matrix) multiplication takes form

$$
\begin{aligned}
\left(x_{1}, x_{2}, x_{3}\right)\left(y_{1}, y_{2}, y_{3}\right) & =\left(x_{1}+y_{1}, x_{2}+y_{2}, x_{3}+y_{3}+x_{1} y_{2}\right) \\
\left(x_{1}, x_{2}, x_{3}\right)^{-1} & =\left(-x_{1},-x_{2},-x_{3}+x_{1} x_{2}\right) .
\end{aligned}
$$

One has

$$
a^{k} b^{l}=b^{l} a^{k} c^{k l}
$$

This allows to present any element under the form $b^{x_{2}} a^{x_{1}} c^{x_{3}}$, because it is easy to verify that the center of $G$ is the infinite cyclic group generated by $c=(0,0,1)$.

Any automorphism $\phi$ of $G$ maps the center isomorphically onto itself. Hence $\phi(c)=c^{ \pm 1}$. Let $\phi$ map the generators as follows:

$$
\left\{\begin{array}{l}
\phi(a)=(k, l, m)=b^{l} a^{k} c^{m} \\
\phi(b)=(p, q, r)=b^{q} a^{p} c^{r} \\
\phi(c)=c^{s}, \text { where } s= \pm 1
\end{array}\right.
$$

Then for an arbitrary element $\left(x_{1}, x_{2}, x_{3}\right) \in G$

$$
\begin{aligned}
& \phi\left(x_{1}, x_{2}, x_{3}\right)=(\phi(b))^{x_{2}}(\phi(a))^{x_{1}}(\phi(c))^{x_{3}} ; \\
& \phi(b)^{x_{2}}=\underbrace{\left(b^{q} a^{p} c^{r}\right)\left(b^{q} a^{p} c^{r}\right) \cdots\left(b^{q} a^{p} c^{r}\right)}_{x_{2}} \quad \text { (with use of }(6), a c=c a, b c=c b) \\
& =b^{q x_{2}} a^{p x_{2}} c^{r x_{2}+p q\left(1+2+\ldots+\left(x_{2}-1\right)\right)}=b^{q x_{2}} a^{p x_{2}} c^{r x_{2}+p q \frac{x_{2}\left(x_{2}-1\right)}{2}} ; \\
& \phi(a)^{x_{1}}=\underbrace{\left(b^{l} a^{k} c^{m}\right)\left(b^{l} a^{k} c^{m}\right) \cdots\left(b^{l} a^{k} c^{m}\right)}_{x_{1}}=b^{l x_{1}} a^{k x_{1}} c^{m x_{1}+k l \frac{x_{1}\left(x_{1}-1\right)}{2}} .
\end{aligned}
$$

Hence,

$$
\begin{gathered}
\phi\left(x_{1}, x_{2}, x_{3}\right)=b^{q x_{2}} a^{p x_{2}} c^{r x_{2}+p q \frac{x_{2}\left(x_{2}-1\right)}{2}} \cdot b^{l x_{1}} a^{k x_{1}} c^{m x_{1}+k l \frac{x_{1}\left(x_{1}-1\right)}{2}} \cdot c^{s x_{3}} \\
=b^{q x_{2}+l x_{1}} a^{p x_{2}+k x_{1}} c^{r x_{2}+m x_{1}+s x_{3}+p q \frac{x_{2}\left(x_{2}-1\right)}{2}+k l \frac{x_{1}\left(x_{1}-1\right)}{2}+p l x_{1} x_{2}} \\
=\left(k x_{1}+p x_{2}, l x_{1}+q x_{2}, m x_{1}+r x_{2}+s x_{3}+p q \frac{x_{2}\left(x_{2}-1\right)}{2}+k l \frac{x_{1}\left(x_{1}-1\right)}{2}+p l x_{1} x_{2}\right) .
\end{gathered}
$$


Then automorphisms of $G$ have to satisfy

$$
\begin{aligned}
& \phi:\left(x_{1}, x_{2}, x_{3}\right) \mapsto \\
& \begin{aligned}
\left(k x_{1}+p x_{2}, l x_{1}+q x_{2}, m x_{1}+r x_{2}+s x_{3}+p q \frac{x_{2}\left(x_{2}-1\right)}{2}+\right. & \left.k l \frac{x_{1}\left(x_{1}-1\right)}{2}+p l x_{1} x_{2}\right), \\
p, q, r, k, l, m & \in \mathbb{Z}, s= \pm 1 .
\end{aligned}
\end{aligned}
$$

Lemma 6.1. A map of the form (7) is an isomorphism of $G$ if and only if $k q=p l+s$.

Proof. Let us prove that the restriction $k q=p l+s$ is necessary and sufficient for $\phi$ of the form (7) to be an endomorphism.

First, let us verify that the first two components of $\phi(x) \phi(y)$ and $\phi(x y)$ coincide for all $k, l, m, p, q, r, s$ :

$$
\begin{aligned}
\phi(x)= & \left(k x_{1}+p x_{2}, l x_{1}+q x_{2}, *\right) ; \\
\phi(y)= & \left(k y_{1}+p y_{2}, l y_{1}+q y_{2}, *\right) ; \\
& \phi(x) \phi(y)=\left(k\left(x_{1}+x_{2}\right)+p\left(x_{2}+y_{2}\right), l\left(x_{1}+y_{1}\right)+q\left(x_{2}+y_{2}\right), *\right) ; \\
x y= & \left(x_{1}+y_{1}, x_{2}+y_{2}, *\right) ; \\
& \phi(x y)=\left(k\left(x_{1}+x_{2}\right)+p\left(x_{2}+y_{2}\right), l\left(x_{1}+y_{1}\right)+q\left(x_{2}+y_{2}\right), *\right) ;
\end{aligned}
$$

Let us verify the coincidence of the last components of $\phi(x) \phi(y)$ and $\phi(x y)$ for $x=$ $\left(x_{1}, x_{2}, x_{3}\right), y=\left(y_{1}, y_{2}, y_{3}\right)$ :

$$
\begin{array}{r}
\phi(x) \phi(y)=\left(k x_{1}+p x_{2}, l x_{1}+q x_{2}, m x_{1}+r x_{2}+s x_{3}+p q \frac{x_{2}\left(x_{2}-1\right)}{2}+k l \frac{x_{1}\left(x_{1}-1\right)}{2}+p l x_{1} x_{2}\right) . \\
\cdot\left(k y_{1}+p y_{2}, l y_{1}+q y_{2}, m y_{1}+r y_{2}+s y_{3}+p q \frac{y_{2}\left(y_{2}-1\right)}{2}+k l \frac{y_{1}\left(y_{1}-1\right)}{2}+p l y_{1} y_{2}\right)= \\
=\left(*, *, m\left(x_{1}+y_{1}\right)+r\left(x_{2}+y_{2}\right)+s\left(x_{3}+y_{3}\right)+A\right),
\end{array}
$$

where

$$
\begin{aligned}
& A=\frac{p q}{2}\left(x_{2}\left(x_{2}-1\right)+y_{2}\left(y_{2}-1\right)\right)+\frac{k l}{2}\left(x_{1}\left(x_{1}-1\right)+y_{1}\left(y_{1}-1\right)\right)+p l\left(x_{1} x_{2}+y_{1} y_{2}\right)+ \\
& +\left(k x_{1}+p x_{2}\right)\left(l y_{1}+q y_{2}\right)= \\
& =\frac{p q}{2}\left(x_{2}^{2}+y_{2}^{2}-x_{2}-y_{2}+2 x_{2} y_{2}\right)+\frac{k l}{2}\left(x_{1}^{2}+y_{1}^{2}-x_{1}-y_{1}+2 x_{1} y_{1}\right)+p l\left(x_{1} x_{2}+y_{1} y_{2}+x_{2} y_{1}\right)+k q x_{1} y_{2} \text {. }
\end{aligned}
$$

On the other hand,

$$
\begin{aligned}
\phi(x y)=\phi\left(x_{1}+y_{1}, x_{2}+y_{2}, x_{3}+y_{3}\right. & \left.+x_{1} y_{2}\right)= \\
& =\left(*, *, m\left(x_{1}+y_{1}\right)+r\left(x_{2}+y_{2}\right)+s\left(x_{3}+y_{3}\right)+B\right),
\end{aligned}
$$


where

$$
\begin{aligned}
B=s x_{1} y_{2}+\frac{p q}{2}\left(\left(x_{2}+y_{2}\right)\left(x_{2}+y_{2}-1\right)\right) & \\
+\frac{k l}{2} & \left(\left(x_{1}+y_{1}\right)\left(x_{1}+y_{1}-1\right)\right)+p l\left(x_{1}+y_{1}\right)\left(x_{2}+y_{2}\right) \\
\quad & s x_{1} y_{2}+\frac{p q}{2}\left(x_{2}^{2}+y_{2}^{2}-x_{2}-y_{2}+2 x_{2} y_{2}\right) \\
& +\frac{k l}{2}\left(x_{1}^{2}+y_{1}^{2}-x_{1}-y_{1}+2 x_{1} y_{1}\right)+p l\left(x_{1} x_{2}+y_{1} x_{2}+y_{2} x_{1}+y_{1} y_{2}\right) .
\end{aligned}
$$

Hence,

$$
\begin{aligned}
& \begin{array}{l}
\phi(x y)=\phi(x) \phi(y) \Leftrightarrow A=B \Leftrightarrow \\
\Leftrightarrow p l\left(x_{1} x_{2}+y_{1} y_{2}+x_{2} y_{1}\right)+k q x_{1} y_{2}=
\end{array} \quad \begin{array}{l}
p l\left(x_{1} x_{2}+y_{1} x_{2}+y_{2} x_{1}+y_{1} y_{2}\right)+s x_{1} x_{2} \Leftrightarrow \\
\Leftrightarrow k q x_{1} y_{2}=p l x_{1} y_{2}+s x_{1} y_{2} \Leftrightarrow k q=p l+s .
\end{array}
\end{aligned}
$$

Let us verify that the same condition $k q=p l+s$ is sufficient for $\phi$ to be bijective. Suppose $p=\left(p_{1}, p_{2}, p_{3}\right) \in G$. Let us find all elements $x$ such that $\phi(x)=p$, i.e.

$$
\left\{\begin{array}{l}
k x_{1}+p x_{2}=p_{1} \\
l x_{1}+q x_{2}=p_{2} \\
F\left(x_{1}, x_{2}\right)+s x_{3}=p_{3} .
\end{array}\right.
$$

Since $k q-p l=s= \pm 1$, this system of equations has a unique solution $\forall p_{1}, p_{2}, p_{3}$. Hence $\phi$ is a bijection.

Lemma 6.2. If $s=1$, then $R(\phi)=\infty$.

Proof. This follows immediately from Lemma 2.2.

Lemma 6.3. For each $N \in \mathbb{N}$ there exists an automorphism $\phi$ of $G$ such that $R(\phi)=2 N$.

Proof. For any automorphism $\phi$, i.e. a map of the form (7) with $k q-p l= \pm 1$, one has

$$
\phi(x)=\left(k x_{1}+p x_{2}, l x_{1}+q x_{2}, s x_{3}+Q_{0}\left(x_{1}, x_{2}\right)\right) .
$$

Let $g=\left(g_{1}, g_{2}, g_{3}\right)$. Then $g^{-1}=\left(-g_{1},-g_{2}, g_{1} g_{2}-g_{3}\right)$ and

$$
\begin{gathered}
\phi\left(g^{-1}\right)=\left(-k g_{1}-p g_{2},-l g_{1}-q g_{2},-s g_{3}+Q_{1}\left(g_{1}, g_{2}\right)\right), \\
g x \phi\left(g^{-1}\right)=\left(g_{1}+x_{1}, g_{2}+x_{2}, g_{3}+x_{3}+g_{1} x_{2}\right)\left(-k g_{1}-p g_{2},-l g_{1}-q g_{2},-s g_{3}+Q_{1}\left(g_{1}, g_{2}\right)\right) \\
=\left(x_{1}+(1-k) g_{1}-p g_{2}, x_{2}-l g_{1}+(1-q) g_{2}, x_{3}+2 g_{3}+Q_{2}\left(g_{1}, g_{2}, x_{1}, x_{2}\right)\right) .
\end{gathered}
$$

Take $k=1-N, p=N, l=1, q=-1$. Then $s=k q-p l=N-1-N=-1$ as in Lemma 6.1. One has

$$
g x \phi\left(g^{-1}\right)=\left(x_{1}+N g_{1}-N g_{2}, x_{2}+2 g_{2}-g_{1}, x_{3}+2 g_{3}+Q_{2}\left(g_{1}, g_{2}, x_{1}, x_{2}\right)\right) .
$$

Denote $f_{1}:=g_{1}-g_{2}, f_{2}:=2 g_{2}-g_{1}, f_{3}:=g_{3}$. Then $g_{1}=f_{2}+2 f_{1}, g_{2}=f_{2}-f_{1}$, and

(9) $x_{\phi}=\left\{g x \phi\left(g^{-1}\right) \mid g \in G\right\}$

$$
=\left\{\left(x_{1}+N f_{1}, x_{2}+f_{2}, x_{3}+2 f_{3}+Q_{3}\left(f_{1}, f_{2}, x_{1}, x_{2}\right)\right) \mid f_{1}, f_{2}, f_{3} \in \mathbb{Z}\right\} .
$$


Hence, if the first components of $x$ and $y$ do not coincide modulo $N$, then $x \nsim y$. Let

$$
H_{r}=\left\{\left(r+f_{1} N, *, *\right) \in G \mid f_{1} \in \mathbb{Z}\right\} .
$$

Then elements of $H_{i}$ are not equivalent to element of $H_{j}$ if $i \neq j, i, j=1, \ldots, N$.

Let us verify that each of $H_{i}$ consists exactly of two $\phi$-conjugacy classes. Let us remind that

$$
Q_{0}\left(x_{1}, x_{2}\right)=m x_{1}+r x_{2}+p q \frac{x_{2}\left(x_{2}-1\right)}{2}+k l \frac{x_{1}\left(x_{1}-1\right)}{2}+p l x_{1} x_{2} .
$$

This is a polynomial in 2 variables. It is easy to see that the parity of its value for some arguments is determined by their values modulo 4 . Returning to the definitions of $Q_{i}$ one can see that all of them enjoy the same property. Let us fix $r \in\{0, \ldots, N-1\}$. Then in accordance with (9) one has

$$
\begin{aligned}
&\{(r, 0,0)\}_{\phi}=\left\{\left(r+N f_{1}, f_{2}, 2 f_{3}+Q_{3}\left(f_{1}, f_{2}, x_{1}, x_{2}\right)\right) \mid f_{1}, f_{2}, f_{3} \in \mathbb{Z}\right\} \\
&= \bigcup_{i, j \in \mathbb{Z}_{4}}\left\{\left(r+N f_{1}, f_{2}, 2 f_{3}+Q_{3}\left(f_{1}, f_{2}, r, 0\right)\right) \mid f_{1} \in 4 \mathbb{Z}+i, f_{2} \in 4 \mathbb{Z}+j, f_{3} \in \mathbb{Z}\right\} \\
&= \bigcup_{i, j \in \mathbb{Z}_{4}}\left\{\left(r+N f_{1}, f_{2}, 2 f_{3}+\left(Q_{3}(i, j, r, 0)\right) \bmod 2\right) \mid f_{1} \in 4 \mathbb{Z}+i, f_{2} \in 4 \mathbb{Z}+j, f_{2} \in \mathbb{Z}\right\} ; \\
&\{(r, 0,1)\}_{\phi}=\bigcup_{i, j \in \mathbb{Z}_{4}}\left\{\left(r+N f_{1}, f_{2}, 2 f_{3}+1+\left(Q_{3}(i, j, r, 0)\right) \quad \bmod 2\right) \mid\right. \\
&\left.f_{1} \in 4 \mathbb{Z}+i, f_{2} \in 4 \mathbb{Z}+j, f_{3} \in \mathbb{Z}\right\} .
\end{aligned}
$$

Hence, each element of $H_{r}$ is equivalent to $(r, 0,0)$ or $(r, 0,1)$. Namely, an element of the form $\left(r+f_{1} N, f_{2}, f_{3}\right)$, where $f_{1}=i(N)$ and $f_{2}=j(N)$, belongs to $\{(r, 0,0)\}_{\phi}$ if and only if $f_{3}$ has the same parity as $Q_{3}(i, j, r, 0)$, and belongs to $\{(r, 0,1)\}_{\phi}$ otherwise.

\section{FINDING OF FIXED REPRESENTATIONS}

Consider the discrete Heisenberg group $G$ as a semi-direct product of $\mathbb{Z}^{2}$ and $\mathbb{Z}$ :

$$
\begin{aligned}
H=\mathbb{Z}^{2} \lambda \mathbb{Z}, \quad \widetilde{\alpha}: \mathbb{Z} & \rightarrow \operatorname{Aut}\left(\mathbb{Z}^{2}\right) ; \\
s & \mapsto \alpha^{s}, \quad \alpha=\left(\begin{array}{ll}
1 & 1 \\
0 & 1
\end{array}\right) .
\end{aligned}
$$

So, $G$ consists of integral triples $((m, k), s)$ with the following multiplication:

$$
((m, k), s) *\left(\left(m^{\prime}, k^{\prime}\right), s^{\prime}\right)=\left((m, k)+\alpha^{s}\left(m^{\prime}, k^{\prime}\right)\right)=\left(\left(m+m^{\prime}, k+k^{\prime}+s m^{\prime}\right), s+s^{\prime}\right) ;
$$

In particular,

$$
((m, k), 0) *((0,0), s)=((m, k), s)
$$

The dual object for $\mathbb{Z}^{2}$ is the torus $\mathbb{T}^{2}$. A pair $\chi=(\xi, \eta) \in \mathbb{T}^{2}$ corresponds to the character $(m, k) \mapsto e^{2 \pi i(m \xi+k \eta)}$. The torus is a right $G$-space for the action

$$
\chi h(m, k)=\chi\left(h *((m, k), 0) * h^{-1}\right) .
$$

The action of $((m, k), s)$ is defined by the formula

$$
(\xi, \eta) \mapsto(\xi, \eta)\left(\begin{array}{ll}
1 & 0 \\
s & 1
\end{array}\right)=(\xi+s \eta, \eta) .
$$

We need the following facts. 
Theorem 7.1 (Glimm). [13, Sect. 9.1] For complete separable metric $H$-space $X$ the following properties are equivalent:

(1) Each $H$-orbit in $X$ is locally closed.

(2) The quotient space $X / H$ is a $T_{0}$-space.

(3) There exists a countable family of $H$-invariant Borel subsets in $X$ separating any two $H$-orbits.

(4) Each H-ergodic Borel measure on $X$ is supported on one of these orbits.

Theorem 7.2 (Mackey). [13, p. 197] If $N$ is a closed commutative normal subgroup of a locally compact group $H$ and an action of $H$ on $\widehat{N}$ satisfies the properties of the Glimm's theorem, then any irreducible representation $\rho$ of $H$ has the form $\operatorname{Ind}(H, Y, \beta)$, where $Y$ is the stabilizer of some point $\chi \in \widehat{N}$ and the restriction of $\beta$ on $N$ is a scalar one and is a multiple of the character $\chi$.

Conversely, [2, Theorem 5, p. 509] implies the following. If $\beta$ is an irreducible representation of $Y$ with the restriction on $N$ being a multiple of a character, then $\operatorname{Ind}(H, Y, \beta)$ is irreducible.

Theorem 7.3. [11, Theorem 3.2, Chapter II] Let $G$ be a locally compact group with countable base acting transitively on a locally compact Hausdorff space $\Gamma$. Let $\gamma$ be an arbitrary point of $\Gamma$ with the stabilizer $H$. Then $H$ is closed and the map $g H \mapsto g \gamma$ is a homeomorphism of $G / H$ onto $\Gamma$.

Hence, for such $G$ and $\Gamma$ each orbit $O$ is homeomorphic to the quotient space of $G$ by the stabilizer of any point of this orbit.

Let us describe finite dimensional irreducible representations of $H$. By the previous paragraph and the Mackey's theorem, all these representations are induced from the stabilizer of some point $\chi=(\xi, \eta) \in \mathbb{T}^{2}$ such that its orbit $\widehat{O}_{\chi}$ is finite. Let it for some $\chi$ consist of $p$ points. The stabilizer of $\chi$ is

$$
Y=\{((m, k), p s) \mid m, k, s \in \mathbb{Z}\}=\mathbb{Z}^{2} \lambda(p \mathbb{Z}) .
$$

By $[2$, Lemma 3, p. 508] the multiplication by the character of $\chi$ is a bijection between the collections of irreducible unitary representations $p \mathbb{Z}$ and $Y=\mathbb{Z}^{2} \lambda(p \mathbb{Z})$.

Hence, any $p$-dimensional irreducible unitary representation of $H(P<\infty)$ can be obtained as a result of the following procedure: 1) choose a point $\chi=(\xi, \eta) \in \mathbb{T}^{2}$ with the orbit of cardinality $p ; 2)$ choose an irreducible representation $\alpha$ of the subgroup $p \mathbb{Z}=\{((0,0), p s) \mid s \in \mathbb{Z}\} ; 3)$ multiply this representation by the character of $\chi$ and obtain a representation $\beta$ of the corresponding subgroup $Y$; 4) form the representation $\rho$ of $H$ induced by $\beta$.

Choose an arbitrary $\alpha \in[0,1)$ and consequently an irreducible representation $\pi$ of the subgroup $(p \mathbb{Z})$ :

$$
\pi((0,0) p s)=e^{2 \pi i s \alpha}
$$

The multiplication by the character $\chi=(\xi, \eta)$ gives rise the following representation $\beta$ of the subgroup $Y$ :

$$
\beta((m, k), p s)=\chi(m, k) e^{2 \pi i s \alpha}=e^{2 \pi i(m \xi+k \eta+s \alpha)} ;
$$

Now we have to form the representation $\rho$ of $H$ induced by $\beta$. For this purpose let us remind the realization of the induced representation in the space of $L^{2}$-functions on $X=Y \backslash H$ (see, e.g. [13, pp. 188-190]) for the case of a discrete group. 
Let $H$ be a discrete group, $Y$ its subgroup, $\beta$ a unitary representation of $Y$ in a Hilbert space $V, X=Y \backslash H$ the corresponding right homogeneous space. Let us fix a map $s: X \rightarrow G$ such that $s(H g) \in H g$. Then the induced representation $\rho$ in the space $L^{2}(X, V)$ is defined by the following formula:

$$
[\rho(h) f](x)=A(h, x) f(x h),
$$

where the operator valued function $A(h, x)$ is defined by

$$
A(h, x)=\beta(y),
$$

while $y \in Y$ is defined by

$$
s(x) h=y s(x h) .
$$

In our case $\beta$ is 1-dimensional and $A(h, x)$ is a complex valued function. One has $Y((m, k), s)=Y((0,0), s \bmod p)$. Hence,

$$
Y \backslash H=\{Y((0,0), 0), \quad Y((0,0), 1), \quad \ldots, \quad Y((0,0), p-1)\}=:\left\{x_{0}, x_{1}, \ldots, x_{p-1}\right\} .
$$

Choose $s: X \rightarrow G$ being

$$
s: Y((m, k), s) \longmapsto((0,0), s \bmod p) .
$$

Now we have for given $x \in X=Y \backslash H$ and $h \in H$ to find $y \in Y$ in accordance with $s(x) h=y s(x h)$. Let $h=((m, k), s), x=Y((0,0), j)$. Then $s(x)=((0,0), j)$;

$$
\begin{gathered}
s(x) h=((0,0), j)((m, k), s)=((m, k+j m), s+j) \\
s(x h)=s(Y((0,0), j)((m, k), s))=s(Y(m, k+j m), s+j)=((0,0),(s+j) \bmod p) .
\end{gathered}
$$

Let the desired $y$ be $y=\left(\left(y_{k}, y_{m}\right), y_{s}\right)$. Then

$$
y s(x h)=\left(\left(y_{k}, y_{m}\right), y_{s}\right)((0,0),(s+j) \bmod p)=\left(\left(y_{m}, k\right), y_{s}+(s+j) \bmod p\right) .
$$

Hence,

$$
y_{m}=m, \quad y_{k}=k+j m, \quad y_{s}=s+j-(s+j) \bmod p .
$$

Denote $[l]_{p}:=l-l \bmod p$. Then $y=\left((m, k+j m),[s+j]_{p}\right)$ and

$$
\begin{gathered}
A(h, x)=\beta(y)=\beta\left((m, k+j m),[s+j]_{p}\right)=e^{2 \pi i\left(m \xi+(k+j m) \eta+\frac{[s+j]_{p}}{p}\right)} \\
=e^{2 \pi i\left(m \xi+(k+j m) \eta+\left[\frac{s+j}{p}\right]\right)},
\end{gathered}
$$

where $[r]$ is the entire part of $r$. Finally, the induced representation $\rho$ in $L^{2}(X)=$ $L^{2}\left(\left\{x_{0}, x_{1}, \ldots, x_{p-1}\right\}\right)$ is given by

$$
\begin{aligned}
{[\rho(h) f](x) } & =A(h, x) f(x h)=e^{2 \pi i\left(m \xi+(k+j m) \eta+\left[\frac{s+j}{p}\right]\right)} f(x h) \\
{[\rho((m, k), s) f]\left(x_{j}\right) } & =e^{2 \pi i\left(m \xi+(k+j m) \eta+\left[\frac{s+j}{p}\right]\right)} f\left(x_{(j+s) \bmod p)}\right) \quad j=\overline{0, p-1} .
\end{aligned}
$$

Let us choose in $L^{2}(X)=L^{2}\left(\left\{x_{0}, x_{1}, \ldots, x_{p-1}\right\}\right)$ the base $\epsilon_{0}, \epsilon_{1}, \ldots, \epsilon_{p-1}$, where $\epsilon_{j}$ is the indicator of a point $x_{j} \in X$. With the respect to this base our representation is defined by

$$
\rho((m, k), s): \epsilon_{j} \mapsto e^{2 \pi i\left(m \xi+(k+j m) \eta+\left[\frac{s+j}{p}\right] \alpha\right)} \epsilon_{(j-s) \bmod p} ; \quad j=\overline{0, p-1} .
$$

So, all finite dimensional irreducible unitary representations of $H$ are of the form (10) with some $\xi, \eta, \alpha \in[0,1)$, and the orbit of $\chi=(\xi, \eta) \in \mathbb{T}^{2}$ consists of $p$ points. The action of $((m, k), s)$ on $\mathbb{T}^{2}$ is given by

$$
(\xi, \eta) \mapsto(\xi+s \eta, \eta)
$$


Hence the orbit of this action has cardinality $p$ iff $\eta$ is an irreducible fraction with denominator $p$.

Let us find the character $\chi_{\rho}$ of the representation (10). The matrix of $\rho((m, k), s)$ is diagonal for $s \equiv 0 \bmod p$ and has zeros on the diagonal for other $s$. Hence, for $s$ non divisible by $p$ one has $\chi_{\rho}((m, k), s)=0$. So,

$$
\chi_{\rho}((m, k), s)=\delta_{s \bmod p}^{0} \sum_{j=0}^{p-1} \exp \left(2 \pi i\left(m \xi+k \eta+j m \eta+\left[\frac{s+j}{p}\right] \alpha\right)\right) .
$$

For $s$ divisible by $p$ and $j \in \overline{0, p-1}$ one has $\left[\frac{s+j}{p}\right]=\frac{s}{p}$. Let us transform the expression:

$$
\chi_{\rho}((m, k), s)=\delta_{s \bmod p}^{0} \exp \left(2 \pi i\left(m \xi+k \eta+\frac{s}{p} \alpha\right)\right) \sum_{j=0}^{p-1} e^{2 \pi i m \eta j} .
$$

To calculate the above sum of $p$ terms of a geometric progression, let us observe that $e^{2 \pi i m \eta}=1$ iff $m \eta \in \mathbb{Z}$, i.e. $m \equiv 0 \bmod p$. Hence,

$$
\sum_{j=0}^{p-1} e^{2 \pi i m \eta j}= \begin{cases}p, & \text { if } m \equiv 0 \bmod p \\ \frac{\exp (2 \pi i m \eta p)-1}{\exp (2 \pi i m \eta)-1} & \text { if } m \neq \equiv 0 \bmod p .\end{cases}
$$

But $\eta p \in \mathbb{Z}$. Hence, $\exp (2 \pi i m \eta p)-1=0$. As a result we obtain

$$
\chi_{\rho}((m, k), s)= \begin{cases}p \cdot e^{2 \pi i\left(m \xi+k \eta+\frac{s}{p} \alpha\right)}, & \text { if } s \equiv 0 \bmod p \text { and } m \equiv 0 \bmod p, \\ 0, & \text { otherwise. }\end{cases}
$$

Consider the following automorphism $\phi$ of $G$.

$$
\phi((m, k), s)=\left(\left(s+m,-k+\frac{m(m-1)}{2}+s m\right), m\right) .
$$

After identification of the representation from the present section and the previous one, we can easily see that $R(\phi)=2$. Let us find implicitly the character of the representation $\rho \phi$.

One has:

$$
\begin{aligned}
& \rho \phi((m, k), s) \epsilon_{j}=\rho\left(\left(s+m,-k+\frac{m(m-1)}{2}+s m\right), m\right) \epsilon_{j}= \\
& \quad=\exp 2 \pi i\left((s+m) \xi+\left(-k+\frac{m(m-1)}{2}+s m+j s+j m\right) \eta+\left[\frac{m+j}{p}\right] \alpha\right) \epsilon_{(j-m) \bmod p} .
\end{aligned}
$$

Analogously calculations above, $\chi_{\rho \phi}$ vanishes on elements with $m \not \equiv 0 \bmod p$, and then we use the fact that $\left[\frac{m+j}{p}\right]=\frac{m}{p}$ if $m \equiv 0 \bmod p$ and $j \in \overline{1, p}$;

$\chi_{\rho \phi}((m, k), s)=\delta_{m \bmod p}^{0} \exp 2 \pi i\left((s+m) \xi+\left(-k+\frac{m(m-1)}{2}+s m\right) \eta+\frac{m}{p} \alpha\right) \sum_{j=0}^{p-1} e^{2 \pi i(s+m) \eta j}$,

and

$$
\sum_{j=0}^{p-1} e^{2 \pi i(s+m) \eta j}= \begin{cases}p, & \text { if }(s+m) \eta \in \mathbb{Z} \text { i.e. if }(s+m) \equiv 0 \bmod p \\ 0, & \text { if }(s+m) \eta \notin \mathbb{Z}\end{cases}
$$


Hence,

$$
\chi_{\rho \phi}((m, k), s)= \begin{cases}p \cdot e^{2 \pi i\left((s+m) \xi+\left(-k+\frac{m(m-1)}{2}+s m\right) \eta+\frac{m}{p} \alpha\right)}, & \text { if } s \text { and } m \equiv 0 \bmod p \\ 0, & \text { otherwise }\end{cases}
$$

Let us find the finite-dimensional fixed points of $\widehat{\phi}$. One of them is the trivial 1dimensional representation.

For finding another, let us write the property of coincidence of characters:

$$
e^{2 \pi i\left((s+m) \xi+\left(-k+\frac{m(m-1)}{2}+s m\right) \eta+\frac{m}{p} \alpha\right)}=e^{2 \pi i\left(m \xi+k \eta+\frac{s}{p} \alpha\right)} \text { for } s, m \equiv 0 \bmod p .
$$

Let $p=2$. Then $\eta=\frac{1}{2}$. Then

$$
e^{2 \pi i\left((s+m) \xi+\frac{1}{2}\left(-k+\frac{m(m-1)}{2}+s m\right)+\frac{m}{2} \alpha\right)}=e^{2 \pi i\left(m \xi+\frac{1}{2} k+\frac{s}{2} \alpha\right)} \text { for even } s, m .
$$

Putting $s=: 2 t, m=: 2 q$ we obtain:

$$
e^{2 \pi i\left((2 t+2 q) \xi+\frac{1}{2}(-k+q(2 q-1)+4 t q)+q \alpha\right)}=e^{2 \pi i\left(2 q \xi+\frac{1}{2} k+t \alpha\right)} \text { for any } s, m \in \mathbb{Z} .
$$

This is equivalent to

$$
\left((2 t+2 q) \xi+\frac{1}{2}(-k+q(2 q-1)+4 t q)+q \alpha\right)-\left(2 q \xi+\frac{1}{2} k+t \alpha\right) \in \mathbb{Z} \text { for any } s, m \in \mathbb{Z} .
$$

After reducing and cancelation of entire summands $q^{2}, 2 t q,-k$ one gets:

$$
2 t \xi-\frac{q}{2}+q \alpha-t \alpha \in \mathbb{Z} \text { for any } s, m \in \mathbb{Z} ; \Leftrightarrow
$$

$$
\Leftrightarrow t(2 \xi-\alpha)+q\left(\alpha-\frac{1}{2}\right) \in \mathbb{Z}, \forall s, m \in \mathbb{Z}
$$

This relation is evidently satisfied for $\alpha=\frac{1}{2}, \xi=\frac{1}{4}$.

So the fixed class representation is two-dimensional. It is defined by (10) with $\alpha=$ $\frac{1}{2}, \xi=\frac{1}{4}, \eta=\frac{1}{2}$, i. e.

$$
\rho_{2}((m, k), s): \epsilon_{j} \mapsto e^{2 \pi i\left(\frac{m}{4}+\frac{k+j m}{2}+\frac{1}{2}\left[\frac{s+j}{2}\right]\right)} \epsilon_{(j-s) \bmod 2} ; \quad j=0,1 .
$$

\section{REFERENCES}

1. J. Arthur and L. Clozel, Simple algebras, base change, and the advanced theory of the trace formula, Princeton University Press, Princeton, NJ, 1989. MR 90m:22041

2. A. O. Barut and R. Raczka, Theory of group representations and applications, second ed., World Scientific Publishing Co., Singapore, 1986. MR 88c:22013

3. J. Dixmier, $C^{*}$-algebras, North-Holland, Amsterdam, 1982.

4. A. Fel'shtyn, Dynamical zeta functions, Nielsen theory and Reidemeister torsion, Mem. Amer. Math. Soc. 147 (2000), no. 699, xii+146. MR 2001a:37031

5. A. Fel'shtyn and E. Troitsky, A twisted Burnside theorem for countable groups and Reidemeister numbers, Proc. Workshop Noncommutative Geometry and Number Theory (Bonn, 2003) (K. Consani, M. Marcolli, and Yu. Manin, eds.), Vieweg, Braunschweig, 2004, (Preprint MPIM2004-65), pp. 000000 .

6. __ Twisted Burnside theorem, Preprint, Max-Planck-Institut für Mathematik, 2005, (submitted to Invent. Math.).

7. A. L. Fel'shtyn, The Reidemeister number of any automorphism of a Gromov hyperbolic group is infinite, Zap. Nauchn. Sem. S.-Peterburg. Otdel. Mat. Inst. Steklov. (POMI) 279 (2001), no. 6 (Geom. i Topol.), 229-240, 250. MR 2002e:20081 
8. D. Gonçalves and P. Wong, Twisted conjugacy classes in exponential growth groups, Bull. London Math. Soc. 35 (2003), no. 2, 261-268. MR 2003j:20054

9. Daciberg L. Gonçalves, The coincidence Reidemeister classes of maps on nilmanifolds, Topol. Methods Nonlinear Anal. 12 (1998), no. 2, 375-386. MR MR1701269 (2000d:55004)

10. A. Grothendieck, Formules de Nielsen-Wecken et de Lefschetz en géométrie algébrique, Séminaire de Géométrie Algébrique du Bois-Marie 1965-66. SGA 5, Lecture Notes in Math., vol. 569, SpringerVerlag, Berlin, 1977, pp. 407-441.

11. Sigurđur Helgason, Differential geometry and symmetric spaces, Pure and Applied Mathematics, Vol. XII, Academic Press, New York, 1962. MR MR0145455 (26 \#2986)

12. B. Jiang, Lectures on Nielsen fixed point theory, Contemp. Math., vol. 14, Amer. Math. Soc., Providence, RI, 1983.

13. A. A. Kirillov, Elements of the theory of representations, Springer-Verlag, Berlin Heidelberg New York, 1976.

14. S. Shokranian, The Selberg-Arthur trace formula, Springer-Verlag, Berlin, 1992, Based on lectures by James Arthur. MR 93j:11029

15. E. Troitsky, Noncommutative Riesz theorem and weak twisted Burnside theorem, Funct. Anal. Appl. 00 (2005), 00-00, (accepted). (Preprint 86, Max-Planck-Institut für Mathematik, 2004).

Fachbereich Mathematik, Emmy-Noether-Campus, Universität Siegen, Walter-FlexStr. 3, D-57068 Siegen, Germany and Instytut Matematyki, Uniwersytet Szczecinski, Ul. Wielkopolska 15, 70-451 Szczecin, Poland

E-mail address: felshtyn@math.uni-siegen.de

URL: http://www.math.uni-siegen.de/felshtyn

Dept. of Mech. and Math., Moscow State University, 119992 GSP-2 Moscow, Russia

E-mail address: indukaev@mail.ru

Dept. of Mech. and Math., Moscow State University, 119992 GSP-2 Moscow, Russia

E-mail address: troitsky@mech.math.msu.su

URL: http://mech.math.msu.su/ ${ }^{\sim}$ troitsky 